Ringner, Markus

[16]

\section{A layered perceptron approach to categorizing cDNA microarray measurements}

Markus Ringner ${ }^{1}$, Carsten Peterson ${ }^{2}$, Sofia Gruvberger², Mårten Fernö ${ }^{2}$, Åke Borg ${ }^{2}$, Javed Khan ${ }^{1}$, J un Wei ${ }^{1}$, Lao Saal ${ }^{1}$, Yidong Chen ${ }^{1}$, Marc Ladanyi ${ }^{3}$, Paul S. Meltzer ${ }^{1}$ \& Jeff M. Trent ${ }^{1}$

${ }^{1}$ Lund University, Lund, Sweden

${ }^{2}$ National Human Genome Research Institute, National Institutes of Health, Bethesda, Maryland, USA

${ }^{3}$ Memorial Sloan-Kettering Cancer Center, New York, New York, USA ibration.
We present a supervised regression model for classifying complementary DNA microarray measurements and a method to rank genes according to their importance for the classification. To allow for a supervised regression model with no overfitting, we reduce the dimensionality of the measured samples (as given by number of genes) by principal component analysis and use the ten dominant components as inputs to multilayered perceptron models. We classify samples using a threefold validation procedure, and the procedure is repeated such that numerous multilayered perceptron models are calibrated. Thus each sample is in many validation sets and all these predictions are used as a committee to classify a sample. The sensitivity of the classification on the different genes is determined by the absolute value of the partial derivative of the output with respect to the gene expressions, averaged over samples and multilayered perceptron models. In this way the genes can be ranked. The method is successfully applied to classify solid tumors in the category of small blue round-cell tumors as well as sporadic breast tumors. In addition, the method correctly classifies additional blind tests and also identifies whether a blind test is different from the disease categories used for cal-

Romagnolo, Donato [17]

\section{Microarray expression profiles in breast cancer MCF-7 cells following exposure to benzo[a]pyrene and its diol epoxide}

\author{
Donato Romagnolo, Brandon Jeffy, David Samuelson
} $\&$ Claire Payne

University of Arizona, Tucson, Arizona, USA

Maintenance of genome integrity in mammalian cells may be compromised by the concomitant accumulation of DNA damage and loss of repair functions. Polycyclic aromatic hydrocarbons are classic DNA-damaging and tumor-promoting agents. Benzo $[a]$ pyrene $(\mathrm{B}[a] \mathrm{P})$, a prototype $\mathrm{PAH}$, induces several genotoxic responses, including cell cycle arrest, oxidative stress and DNA adduct formation. Exposure of MCF-7 cells to $\mathrm{B}[a] \mathrm{P}(1-5 \mu \mathrm{M})$ and the $\mathrm{B}[a] \mathrm{P}$ diol epoxide (BPDE) (50-100 nM) induced transient S-phase arrest, which was followed by accumulation in $\mathrm{G} 2$ and $\mathrm{M}$ and $70 \%$ loss of cell viability. In cells treated with $\mathrm{B}[a] \mathrm{P}$ and $\mathrm{BPDE}$, we observed segregation of nucleolar material and a drastic reduction in the number of ribosomal fibrillar centers. To identify the expression profile in cells treated with $\mathrm{B}[a] \mathrm{P}$ or $\mathrm{BPDE}$, we analyzed the levels of expression of 1,152 selected genes using a GeneMAP CancerArray in control and treated cells. Changes in gene expression were evaluated twice in duplicate $(n=4)$. Compared with the expression levels in untreated cells, transcripts that were upregulated by both $\mathrm{B}[a] \mathrm{P}$ and BPDE included members of the cytochrome P450, AP-1 (Fra-1, Fra-2) and glutathione $S$-transferase families; PARP; and G1/S-specific cyclins. In contrast, tran- script levels for BRCA-1, G2/M cyclins, bcl-2, junB and fosB were downregulated in cells treated with $\mathrm{B}[a] \mathrm{P}$ or BPDE. Treatment with the metabolite BPDE tended to elicit a stronger response than treatment with $\mathrm{B}[a] \mathrm{P}$. Analysis of expression profiles by microarray may be useful to understand the interactions among overlapping pathways and assess exposure to environmental carcinogens.

Sakamoto, Kathleen

[18]

\section{Protechials: A novel approach to pharmacological inhibition of protein function}

Kathleen Sakamoto ${ }^{1,2}$, Raymond Deshaies $^{2} \&$ Craig Crews $^{3}$

${ }^{1}$ Department of Pediatrics and Pathology, University of California Los Angeles School of Medicine, Los Angeles, California, USA

${ }^{2}$ Howard Hughes Medical Institute and Division of Biology, California Institute of Technology, Pasadena, California, USA

${ }^{3}$ Department of Molecular, Cellular, and Developmental Biology Yale University, New Haven, Connecticut, USA

A central goal of the pharmaceutical and biotechnology industries is to identify small, stable, cell-permeable molecules capable of inhibiting the activity of cellular proteins that contribute to cancer. We propose a general method for developing inhibitors that exploits the unique characteristics of the ubiquitin-dependent proteolytic system of eukaryotic cells. The ultimate purpose of our research is to identify a cell-permeable molecule that binds to the substrate-docking site of a ubiquitin ligase. By covalently linking this molecule to other compounds that bind specific cellular proteins, we intend to develop a new class of drugs that can trigger the destruction of any protein in eukaryotic cells for which there exists a small, cell-permeable ligand. Our goal is to develop an experimental system to prove that this new concept for drug design is experimentally feasible. To this end, we have used an IkBa phosphopeptide that binds with high affinity to the substrate-docking domain of the ubiquitin ligase $b \operatorname{TrCP}$. We have chemically linked this peptide to the fungal metabolite fumagillin, which binds specifically to the cellular enzyme methionine aminopeptidase-2 (MetAP-2). Our results indicate that the peptide-fumagillin chimera tethered the enzyme to $\mathrm{bTrCP}$ and targeted it for ubiquitination and degradation. We hope to expand this approach to develop new strategies to treat cancer.

Salceda, Susana

[19]

\section{Identification of differentially expressed genes in breast cancer}

Susana Salceda, Carrie Drumright, Anthony DiEgidio, Brandon Liang, Ping Hu, Yongming Sun, Vu Viet Hoang, Andrei Munteanu, Herve Recipon, Robert Cafferkey \& Roberto A. Macina

diaDexus, Inc., 3303 Octavius Drive, Santa Clara, California 95054, USA

Breast cancer is the most prevalent cancer, and it ranks second among the causes of cancer deaths in women in the United States. Analysis of differentially expressed genes in breast cancer could help in identifying potential diagnostic markers. We used complementary DNA database mining and the polymerase chain reaction-based suppression subtractive hybridization method (Clontech) to identify differentially expressed genes. Mining the cDNA database, we identified genes or expressed sequence tags (ESTs) that showed breast tissue specificity and upregulation in cancer. Simultaneously we sequenced 10,000 clones for each of seven subtracted libraries prepared using different approaches. A set of new ESTs, with no 